\title{
Aharonov-Bohm effect and plasma oscillations in superconducting tubes and rings.
}

\author{
E. N. Bogachek, I. A. Romanovsky, and Uzi Landman \\ School of Physics, Georgia Institute of Technology, Atlanta, Georgia 30332-0430
}

(Dated: April 20, 2021)

\begin{abstract}
Low frequency plasma oscillations in superconducting tubes are considered. The emergence of two different dimensionality regimes of plasma oscillations in tubes, exhibiting a crossover from one-dimensional to two-dimensional behavior, depending on whether $k R \ll 1$ or $k R \gg 1$, where $k$ is the plasmon wave vector and $R$ is the radius of the tube, is discussed. The Aharonov-Bohm effect pertaining to plasma oscillations in superconducting tubes and rings, resulting in an oscillatory behavior of the plasmon frequency as a function of the magnetic flux, with a flux quantum period $h c / 2 e$ (analog of the Little-Parks effect), is studied. The amplitude of the oscillations is proportional to $(\xi / R)^{2}$, where $\xi$ is the superconducting coherence length.
\end{abstract}

PACS numbers: $73.20 . \mathrm{Mf}, 74.78 .-\mathrm{w}, 74.25 . \mathrm{Ha}$ 


\section{INTRODUCTION}

Collective excitations of charge density, so called plasma oscillations, in small lowdimensional superconducting structures have been a topic of great interest for a long time. There are two types of collective excitations in superconductors. One type is the so-called Carlson-Goldman mode [1]. In this mode the superconducting current oscillations are balanced by the current of the normal electrons, and the charge densities produced by the superconducting and normal electrons are mutually compensated. This mode occurs only for temperatures that are very close to the critical temperature $T_{c}$ [2, 3, 4]. The other type of collective excitations are plasma oscillations which are similar to plasma oscillations in normal metals. Unlike plasma oscillations in normal metals, those occurring in superconductors cannot exist in bulk superconducting samples because the typical frequencies of plasma oscillations in the bulk $\left(10^{16} \mathrm{~Hz}\right)$ are far above the superconducting gap $\Delta$. However, in small systems like superconducting wires, thin films, and tubes, the dispersion relation of plasmons has a sound-like (acoustic) character. In what follows we will consider only acoustic type plasma excitations. We are interested in plasma oscillations that do not break the Cooper pairs i.e. oscillations with frequencies $\omega<2 \Delta / \hbar$.

The existence of such (acoustic) plasmons in superconductors was predicted rather early by Kulik [5], who considered plasma excitations for two geometries: a thin infinite solid wire (essentially one-dimensional, 1D) and a thin infinite film (essentially two-dimensional, 2D). It was found that the dispersion relation for the wire is a linear function of the wave vector $k$ along the wire, whereas for the infinite thin film the frequency of the plasmons is proportional to the square root of the wave vector.

Formally, plasmons in low dimensional superconductors with linear and square root dispersion are similar to those in normal conductors [6, 7, [8], but unlike the latter they have lower frequencies and decay rates. Such "superconducting plasmons" were theoretically analyzed later [9] and they were subsequently observed experimentally in superconducting films [10, 11] and wires [12].

The high sensitivity of non-simply connected superconducting systems (cylinders and rings) to weak magnetic fields is well known, and it is manifested by effects such as flux, or fluxoid, quantization [13], and the Little-Parks effect (oscillations of the critical temperature as a function of the magnetic flux with a period determined by the superconducting flux quantum $h c / 2 e[14$, 15]). Both of these effects may be considered as manifestations of the Aharonov-Bohm (AB) effect [16] (see e.g. a review in [17]). Consequently, it is of interest to investigate possible manifestations of the $\mathrm{AB}$ effect in plasma oscillations in superconductors [18].

In this paper we study plasma oscillations in a superconducting tube with an arbitrary radius $R$ and identify two dimensionality regimes (1D and 2D) of the plasmon dispersion relation and the crossover between them, depending on the magnitude of $k R$ (see Ref.[19]). We demonstrate that the $\mathrm{AB}$ effect, pertaining to plasmons in superconducting tubes and rings, is expressed as oscillations of the plasmon frequencies as a function of the magnetic flux with a universal period of $h c / 2 e$ (the flux quantum of a Cooper pair).

The paper is organized as follows. In Sec. II we discuss the formalism used to describe plasma oscillations in superconducting tubes and calculate their dispersion relations. In Sec. III we use these results to study the behavior of plasmons in tubes and rings placed in a magnetic field. We summarize our results in Sec. IV. 


\section{THE DISPERSION RELATION FOR PLASMA OSCILLATIONS IN A HOL- LOW SUPERCONDUCTING CYLINDER}

In this section we study the propagation of plasma waves in a hollow superconducting cylinder (tube). Let us take the symmetry axis of the cylinder as the $z$ axis of a cylindrical coordinate system and let $\mathbf{r}=(r, \theta)$, be the radius-vector perpendicular to the $z$ axis. For simplicity we assume that the width of the wall of the cylinder, $d$, is much smaller than its radius $R$ (Fig. 1a). Since the motion of charge carriers is restricted to be within the material of the cylinder the charge and current densities can be written in the form

$$
\mathbf{j}=\mathbf{j}_{2} \delta(r-R), \quad \rho=\rho_{2} \delta(r-R),
$$

where $\delta(r-R)$ is the Dirac delta function and $\mathbf{j}_{2}$ and $\rho_{2}$ are the two-dimensional (areal) current and charge density, respectively. For the sake of brevity we omit the subscript 2 later.

Both the current flows and the uncompensated charges will produce electric and magnetic fields around the cylinder. The Fourier components of the field potentials, $\tilde{\varphi}$ and $\tilde{A}$, and those of the surface charge $\tilde{\rho}$ and the current density $\tilde{\mathbf{j}}\left(\tilde{\mathbf{j}}=\left(\tilde{j}_{z}, \tilde{j}_{\theta}\right)\right.$, where $\tilde{j}_{z}$ is the component of the current along the $z$ axis, and $\tilde{j}_{\theta}$ is the circular component) corresponding to the frequency $(\omega)$, the longitudinal wave vector $(k)$, and the circular mode number $(m)$, satisfy the Maxwell equations

$$
\begin{gathered}
\frac{1}{r} \frac{\partial}{\partial r}\left(r \frac{\partial \tilde{\varphi}}{\partial r}\right)-\left[\kappa^{2}+\frac{m^{2}}{r^{2}}\right] \tilde{\varphi}=-4 \pi \tilde{\rho} \delta(r-R), \\
\frac{1}{r} \frac{\partial}{\partial r}\left(r \frac{\partial \tilde{\mathbf{A}}}{\partial r}\right)-\left[\kappa^{2}+\frac{m^{2}}{r^{2}}\right] \tilde{\mathbf{A}}=-\frac{4 \pi}{c} \tilde{\mathbf{j}} \delta(r-R),
\end{gathered}
$$

where $\kappa=\sqrt{k^{2}-(\omega / c)^{2}}$ is the modified wave vector that takes into account retardation effects; here and elsewhere in the paper a superscript " " (for example $\tilde{\varphi}$ ) denotes a Fourier transformed quantity. From the requirement that the potentials $\tilde{\mathbf{A}}$ and $\tilde{\varphi}$ must be continuous and finite everywhere, one readily derives the following expressions for the fields on the surface

$$
\left\{\begin{array}{c}
\tilde{\varphi}=-4 \pi \tilde{\rho} R I_{m}(\kappa R) K_{m}(\kappa R), \\
\tilde{\mathbf{A}}=-\frac{4 \pi}{c} \tilde{\mathbf{j}} R I_{m}(\kappa R) K_{m}(\kappa R) .
\end{array}\right.
$$

The electric fields acting on the superconducting and normal electrons inside the cylinder can be written as

$$
\begin{gathered}
\tilde{E}_{z}=4 \pi i\left(\frac{\omega}{c^{2}} \tilde{j}^{z}-k \tilde{\rho}\right) R I_{m}(\kappa R) K_{m}(\kappa R), \\
\tilde{E}_{\theta}=4 \pi i\left(\frac{\omega}{c^{2}} \tilde{j}^{\theta}-\frac{m}{R} \tilde{\rho}\right) R I_{m}(\kappa R) K_{m}(\kappa R),
\end{gathered}
$$

where $I_{m}(x)$ and $K_{m}(x)$ are the modified Bessel functions of the first and second order, respectively.

Our discussion so far was general. To describe the superconducting regime we adopt a simple two-fluid phenomenological model [20] with a nonlinear superconducting term. We 
assume that the system is almost in a stationary state. For the description of the superconducting component we use the time-independent Ginzburg-Landau equation. It was shown rather early by Bardeen [21], that for clean metals (close to $T_{c}$ ) the two fluid model can be derived directly from the BCS theory. Subsequently it was also shown that the two-fluid model can also be successfully used for dirty metals [22]. The two-fluid model assumes that electrons are locally in thermodynamic equilibrium. In the case of low energy small amplitude long-wavelength collective excitations, like acoustic plasma waves, any gradients in the velocities or densities are sufficiently small, and the equation of motion of the superconducting electrons takes the form as for classically behaving particles.

The electrons of the superconducting component of our two-fluid model move without dissipation, whereas the electrons of the normal fluid dissipate energy. The parameter that characterizes dissipation of the normal fluid $\tau$ is the average time between collisions of the normal electrons in the metal. Unless this collision time is very small there is no significant difference between the normal and superconducting electrons since both contribute to the plasma oscillations. The situation is very different, however, for small collision times $\omega \tau \ll 1$. In this case the normal carriers almost do not participate in the plasma oscillations (see discussion in Sec. IV).

Let us assume that the thickness $d$ of the walls of the hollow cylinder (tube) is smaller than both the superconducting coherence length $\xi$, and the London penetration depth $\delta$, $(d \ll \delta, \xi)$. These assumptions allow us to treat the amplitude of the order parameter $\Delta$ in the cylinder as a constant and the current density (in the dirty superconductor) can be written (from consideration of the time-independent Ginzburg-Landau equation) in the form (see e.g. [23])

$$
\mathbf{j}=e N_{s}^{e f f} \mathbf{v}+\sigma_{n} \mathbf{E}, \quad N_{s}^{e f f}=N_{s}\left(1-\frac{v^{2}}{v_{c}^{2}}\right)
$$

and $N_{s}$ is the concentration of the superconducting electrons, $\mathbf{v}=\left(v_{z}, v_{\theta}\right)$ is their velocity which is a two component vector: $v_{z}$ is the velocity along the tube, $v_{\theta}$ is the velocity in the plane perpendicular to the axis of the tube $\left(v^{2}=v_{z}^{2}+v_{\theta}^{2}\right), v_{c}$ is the critical velocity, $\sigma_{n}$ is the normal conductivity, and $E$ is the external electric field. $N_{s}^{e f f}$ is the "effective concentration" of the superconducting electrons in the film, which depends on the velocity $v$. The first term in the equation for the above equation for $\mathbf{j}$ describes the supercurrent, whereas the second term describes the current due to the normal electrons and it accounts for all the dissipation processes in our system.

The relationship between the current and the velocity of the superconducting electrons is nonlinear (see Eq.7). When the amplitude of the velocity oscillations in the plasma wave is much smaller than the critical velocity $v_{c}$ we can linearize this relationship about the homogeneous solution $\mathbf{u}$

$$
\mathbf{v}=\mathbf{u}+\delta \mathbf{v}
$$

where $\mathbf{u}=\left(u_{z}, u_{\theta}\right)$ with $u_{z}=$ const and $u_{\theta}=$ const. The uniform homogeneous background current through the tube has two components: normal and superconducting. We assume that the superconductor is very dirty and therefore the normal conductance of the tube is very small. Because of the very small normal conductance the normal component of the constant background current is much smaller than the total background current and we neglect the voltage drop due to the normal current through the tube. In general however, if 
one takes into account the voltage due to the normal current, when considering the currents very close to the critical one, superconducting states with a uniform time-independent order parameter may become unstable toward small perturbations and the system may become normal or develop a time-dependent superconducting state (see e.g. [24], [25]). We assume here that we are sufficiently far from the critical current to assure that such transitions do not occur in the system under consideration.

Substituting the continuity equation and the equation of motion written in the Fourier representation

$$
\tilde{\rho}=\frac{m}{\omega R} \tilde{j}^{\theta}+\frac{k}{\omega} \tilde{j}^{z}, \quad \tilde{\mathbf{v}}=-i \frac{e}{\omega m_{e}} \tilde{\mathbf{E}}
$$

into (5) and (6) we derive the relations between the currents and the velocities of the electrons in the superconductor

$$
\left(\begin{array}{c}
\tilde{v}_{z} \\
\tilde{v}_{\theta}
\end{array}\right)=\left(\begin{array}{cc}
a_{z z} & a_{z \theta} \\
a_{\theta z} & a_{\theta \theta}
\end{array}\right)\left(\begin{array}{c}
\tilde{j}_{z} \\
\tilde{j}_{\theta}
\end{array}\right)
$$

where

$$
\begin{array}{r}
a_{z z}=\frac{4 \pi e R}{m_{e} \omega^{2}}\left(k^{2}-\frac{\omega^{2}}{c^{2}}\right) I_{m}(\kappa R) K_{m}(\kappa R), \\
a_{\theta \theta}=\frac{4 \pi e R}{m_{e} \omega^{2}}\left(\frac{m^{2}}{R^{2}}-\frac{\omega^{2}}{c^{2}}\right) I_{m}(\kappa R) K_{m}(\kappa R), \\
a_{\theta z}=a_{z \theta}=\frac{4 \pi e k m}{m_{e} \omega^{2}} I_{m}(\kappa R) K_{m}(\kappa R) .
\end{array}
$$

After Fourier transformation, the relation between the perturbations of the velocity $\delta \mathbf{v}=$ $\left(\delta v_{z}, \delta v_{\theta}\right)$ and the current density $\delta \mathbf{j}=\left(\delta j_{z}, \delta j_{\theta}\right)$ can be written as

$$
\left(\begin{array}{c}
\delta \tilde{j}_{z} \\
\delta \tilde{j}_{\theta}
\end{array}\right)=\left(\begin{array}{cc}
b_{z z} & b_{z \theta} \\
b_{\theta z} & b_{\theta \theta}
\end{array}\right)\left(\begin{array}{c}
\delta \tilde{v}_{z} \\
\delta \tilde{v}_{\theta}
\end{array}\right)
$$

with

$$
\begin{gathered}
b_{z z}=N_{s} e d\left(1-\frac{u_{\theta}^{2}+3 u_{z}^{2}}{v_{c}^{2}}\right)+i \omega \sigma_{n} \frac{m_{e} d}{e}, \\
b_{\theta \theta}=N_{s} e d\left(1-\frac{u_{z}^{2}+3 u_{\theta}^{2}}{v_{c}^{2}}\right)+i \omega \sigma_{n} \frac{m_{e} d}{e}, \\
b_{z \theta}=b_{z \theta}=2 N_{s} e d \frac{u_{z} u_{\theta}}{v_{c}^{2}} .
\end{gathered}
$$

Combining equations (10) and (14) we get a linear algebraic system of equations

$$
\left(\begin{array}{cc}
a_{z z} & a_{z \theta} \\
a_{\theta z} & a_{\theta \theta}
\end{array}\right)\left(\begin{array}{cc}
b_{z z} & b_{z \theta} \\
b_{\theta z} & b_{\theta \theta}
\end{array}\right)\left(\begin{array}{c}
\delta \tilde{v}_{z} \\
\delta \tilde{v}_{\theta}
\end{array}\right)=\left(\begin{array}{c}
\delta \tilde{v}_{z} \\
\delta \tilde{v}_{\theta}
\end{array}\right) .
$$


In matrix notation the above can be written as

$$
(\tilde{\mathbf{A}} \tilde{\mathbf{B}}-\tilde{\mathbf{I}}) \delta \tilde{\mathbf{v}}=0
$$

where $\tilde{\mathbf{I}}$ is the identity matrix.

This system of equations has nontrivial solutions if the determinant of the matrix $\tilde{\mathbf{C}}=$ $\tilde{\mathbf{A}} \tilde{\mathbf{B}}-\tilde{\mathbf{I}}$ is zero. The coefficients of matrices $\tilde{\mathbf{A}}$ and $\tilde{\mathbf{B}}$ are functions of $k, m$ and $\omega$, and the condition $\mathcal{D}(k, m, \omega)=\operatorname{det}(\tilde{\mathbf{A}} \tilde{\mathbf{B}}-\tilde{\mathbf{I}})=0$ gives implicitly the desired dispersion relation for the plasma excitations in the cylinder. Since the resulting expression is rather cumbersome we do not reproduce it here but represent the result graphically in Fig 2 (for $\sigma_{n}=0$ ).

Assuming that the normal conductivity of the material of the superconductor is zero, the general relation for the frequency of the plasmons can be written approximately in the form

$$
\begin{aligned}
\omega^{2}=\omega_{s}^{2}\left(\frac{d}{R}\right)\left[k^{2} R^{2}\right. & \left(1-\frac{3 u_{z}^{2}+u_{\theta}^{2}}{v_{c}^{2}}\right)+ \\
& \left.+m^{2}\left(1-\frac{u_{z}^{2}+3 u_{\theta}^{2}}{v_{c}^{2}}\right)-4 m k R \frac{u_{z} u_{\theta}}{v_{c}^{2}}\right] I_{m}(\kappa R) K_{m}(\kappa R),
\end{aligned}
$$

where $\omega_{s}=\omega_{0} \sqrt{N_{s} / N}, N$ is total concentration of electrons (normal and superconducting) and $\omega_{0}=\sqrt{4 \pi e^{2} N / m_{e}}$ is a frequency of plasma oscillations in a bulk normal metal. In deriving Eq.20 we neglected terms of the order of $\omega_{s}^{2} R d / c^{2} \ll 1$, which are related to relativistic retardation effects and are small for tubes of practical sizes.

There are two important cases for which the dispersion relation for the plasma oscillations can be written explicitly in a simple form. These are: (i) the limiting case of plasma oscillations in an infinite superconducting thin wire (1D case), and (ii) plasma oscillations in a thin superconducting thin film (2D case).

In the first case (i), the radius $R$ should be reduced to the limit when the cylinder becomes a thin thread without a hole inside. In this limit $(R=d)$ one gets for the circular mode with $m=0$, when $u_{\theta}=0$

$$
\omega=\omega_{s} k d \sqrt{\left(1-\frac{3 u^{2}}{v_{c}^{2}}\right) \ln \left(\frac{2}{\gamma k d}\right)},
$$

where we used asymptotic expressions for the modified Bessel functions for small values of the arguments, $x \ll 1\left(I_{0}(x) \rightarrow 1\right.$ and $\left.K_{0}(x) \rightarrow-\ln (\gamma x / 2)\right)$, and the constant $\gamma=$ $\exp (C) \approx 1.781$ is the exponent of Euler's constant. Such a linear dispersion relation is typical for one-dimensional conductors.

To obtain the dispersion relation for the thin superconducting thin film (case(ii)) one should take the large radius limit for the cylinder $(k R \gg 1)$. In this case we obtain a square root dispersion relation $\left(u_{\theta}=0\right)$

$$
\omega^{2}=\frac{\omega_{s}^{2} k d}{2}\left(1-\frac{3 u^{2}}{v_{c}^{2}}\right) .
$$

Note that Eqs. (21) and (22) reproduce the expressions derived earlier by Kulik for superconducting thin wires and films ( see Eqs. (14) and (17) in Ref. [5], where the thin wire is 
referred to as a "filament"). Similarly, using asymptotic expressions for the modified Bessel functions of high orders (i.e. $m \gg 1$ ) we obtain for $k R \rightarrow 0$ the expression $\left(u_{\theta}=0\right)$

$$
\omega^{2}=\frac{\omega_{s}^{2} m d}{2 R}\left(1-\frac{u^{2}}{v_{c}^{2}}\right) .
$$

This expression shows that for large $m$ the plasmon frequency $\omega$ is proportional to the $\sqrt{m}$, where $m$ is the circular mode number.

An interesting property that emerges from the expression given in Eq. (20) is that the frequency of the plasma oscillation can be decreased by passing an electric current through the tube. By increasing the current (i.e. increasing the velocities $u_{z}$ and $u_{\theta}$ in Eq. (20)) and bringing it close to the critical current in the film, $j_{\max }$, one can lower the frequency of the plasmons and cause it to take values that lie below the energy gap. This lowering can be achieved for a range of wave vectors that is large enough to allow observation of a dimensionality crossover from a $1 \mathrm{D}(k R \ll 1)$ to a $2 \mathrm{D}(k R \gg 1)$ behavior [19]; see Fig. 2 where we display the plasmon frequency as a function of the dimensionless radius $k R$, for several values of the current $I$ (expressed in terms of $\eta=I / I_{c}$ ) through the tube $\left(I_{1}<I_{2}<I_{3}\right)$. Note that higher currents through the tube correspond to lower frequencies of the plasmons. The observed decrease of the plasmon frequency originates from the fact that, according to Eq. (17), increasing the current along the tube involves an increase in the velocities (i.e. $u_{z}$ ) of the superconducting electrons in the tube which in turn causes an effective decrease in the concentration of the superconducting electrons that participate in plasma oscillations and determine the plasma frequency.

\section{SUPERCONDUCTING TUBES AND RINGS IN AN EXTERNAL MAG- NETIC FIELD}

In this section we analyze the influence of a weak magnetic field on the propagation of plasma excitations in superconducting microstructures. We consider plasmons in two geometries: tubes and rings.

\section{A. Tubes}

Let us consider the situation when the superconducting microcylinder is placed in a longitudinal magnetic field $H$ (Fig. 1a). We assume that the magnetic field is parallel to the symmetry axis of the cylinder and that it is weak enough such that the system remains superconducting. This geometry allows observation of several interesting effects, such as quantization of the magnetic flux through a hole in the cylinder, and periodic dependence of the critical temperature. One may also inquire about the influence of the magnetic field on the dispersion relation of the plasma oscillations.

Since the cylinder wall is made from a very thin film (we take the width of the wall of the cylinder to be smaller than both the London penetration length $\lambda$, the coherence length

$\xi$, and the magnetic length $l_{H}=\sqrt{\Phi_{0} / H}$ ), the amplitude of the superconducting order parameter $|\Psi|$ is constant across the wall of the cylinder. The magnetic field penetrates into the wall and the flux is not quantized. The quantity that is quantized in this case is the total change of the phase of the order parameter (so called 'fluxoid') [23]. Due to quantization of 
the fluxoid, the average circular velocity $u_{\theta}$ of the electrons in the thin-walled cylinder is a periodic function of the magnetic flux

$$
u_{\theta}=\min _{n}\left[\frac{\hbar}{m_{e} R}\left(n-\frac{\Phi}{\Phi_{0}}\right)\right],
$$

where $\Phi=\pi R^{2} H$ is the magnetic flux through the cylinder and $\Phi_{0}=\pi \hbar c / e$ is the superconducting quantum of the magnetic flux. The notation $\min _{n}[\ldots]$ in the above formula denotes that for a given value of the flux $\Phi$ one should take a value of $n$, which minimizes the velocity $u_{\theta}$. One should remember, however, that in order to observe the above quantization phenomenon the radius of the cylinder should be not too small, in order to allow that even weak fields, that do not destroy the superconductivity, may create fluxes $\Phi$ of the order of the flux quantum. Note that since the wall thickness $d$ is small, Little-Parks oscillations $\left(\pi R^{2} H>\Phi_{0}\right)$ might be observed in samples with $\pi R^{2} H_{c}<\Phi_{0}$, where $H_{c}$ is the critical magnetic field for the bulk [26]. Inserting the above expression for the velocity $u_{\theta}$ into Eq.(20) and considering for simplicity only the case with $m=0$, we obtain

$$
\omega^{2}=\frac{4 \pi e^{2} N_{s}^{e f f}}{m_{e}} k^{2} R d I_{0}(\kappa R) K_{0}(\kappa R),
$$

where

$$
N_{s}^{e f f}=N_{s}\left(1-3 \frac{u_{z}^{2}}{v_{c}^{2}}-\frac{\hbar^{2}}{m_{e}^{2} R^{2} v_{c}^{2}} \min _{n}\left[\left(n-\frac{\Phi}{\Phi_{0}}\right)^{2}\right]\right) .
$$

From Eq.(25) we can conclude that the frequencies $\omega$ of the plasmons, as well as their velocities, demonstrate an Aharonov-Bohm (AB) behavior i.e. a periodic dependence on the magnetic flux with the fundamental period $\Phi_{0}$ (Fig. [3). The amplitude of the oscillations of the frequency for a cylinder with a large radius $(R>\xi)$ is approximately proportional to $(\xi / R)^{2}$, where $\xi$ is the coherence length of the superconductor.

To illustrate the above analysis, we show in Fig. 3 the behavior of the plasmon frequencies, $\omega$, in a superconducting tube as a function of the dimensionless flux $\Phi / \Phi_{0}$. In Fig. 3) (a) we display $\omega$ for various values of the current, characterized by the parameter $\eta=I / I_{c}$ where $I_{c}$ is the critical current, for a fixed temperature, and in Fig. 3(b) we show $\omega$ for several temperatures, while keeping the current at a constant value $I=0.1 I_{c}$. The numerical results presented in Fig. 3 were obtained from the transcendental equation (25) (where the arguments of the modified Bessel functions depend on $\kappa=\sqrt{k_{z}^{2}-\omega^{2} / c^{2}}$ ). Note (see Fig. 3(a)) that the amplitudes of the AB oscillations of the frequencies are larger for higher currents through the tube, while at the same time the absolute values of the frequencies decrease for larger currents. The latter decrease of the plasmon frequency for higher currents was discussed in the context of Fig. 2 at the end of Sec. II. The increase of the amplitude of the oscillation of the plasmon frequencies for larger currents is due to the nonlinear dependence of the plasmon frequency on the effective concentration of superconducting electrons $\left(\omega \sim \sqrt{N_{s}^{e f f}}\right)$ in Eq. (25). As a result of this nonlinearity, for a lower concentration of the superconducting electrons (caused by the flow of current, or due to higher temperature, with either of these resulting in effective lowering of the concentration of electrons which may participate in collective plasma oscillations) a given change in the concentration due to magnetically induced circular currents will yield a relatively larger variation of the plasma frequency. The decrease of the plasmon frequency as the temperature is increased is illustrated in Fig. 3(b). 


\section{B. Rings}

We consider here a superconducting ring made from a wire of diameter $d$, with the radius of the ring, $R$, being much larger than $d$ (i.e. $R \gg d$, see Fig.1b). For small currents in the ring we can neglect interactions between different parts of the wire and consider the ring as a straight superconducting wire with imposed periodic boundary conditions. Let us fix a point on the ring as the origin of a local coordinate system and let $x$ be the coordinate along the wire and $\mathbf{r}$ the coordinate perpendicular to the wire. The relation between the Fourier components of small perturbations of the charge density and the electrostatic potential can be written as

$$
\delta \tilde{\phi}(\nu, \omega)=\delta \tilde{\rho}_{1 D}(\nu, \omega) \ln \left(\frac{\nu^{2}+\nu_{c}^{2}}{\nu^{2}}\right)
$$

where $\nu=1,2,3, \ldots$ is now the discrete dimensionless wave number of the plasma oscillations along the ring (which is related to the quantized wave vector $k$ along the ring as $\nu=k R$ ), and $\nu_{c}$ is the cut-off parameter, $\nu_{c} \sim R / d$, which arises because of the finite diameter of the wire from which the ring is made $\left(\nu \ll \nu_{c}\right)$. Using the relation between the scalar potential and the electric field $\tilde{E}(\nu, \omega)=i \nu \tilde{\phi}(\nu, \omega) / R$, the equation of motion $\omega \tilde{v}(\nu, \omega)=-i e \tilde{E}(\nu, \omega) / m_{e}$ and the continuity equation $k \tilde{I}(\nu, \omega)=\omega \tilde{\rho}_{1 D}(\nu, \omega) R$ written in the Fourier representation, we find the expression that connects the perturbation of the carrier velocity with the perturbation of the current

$$
\delta \tilde{v}(\nu, \omega)=\delta \tilde{I}(\nu, \omega) \frac{e \nu^{2}}{\omega^{2} m_{e} R^{2}} \ln \left(\frac{\nu^{2}+\nu_{c}^{2}}{\nu^{2}}\right) .
$$

At this point we use again a linearized form of Eq. (7)

$$
\delta I(\nu, \omega)=e N_{s} S\left(1-\frac{3 u^{2}}{v_{c}^{2}}\right) \delta v(\nu, \omega)
$$

where $S=\pi d^{2} / 4$ is the cross section of the wire making up the ring.

Following the same arguments as those used by us in our discussion of the cylindrical system under the influence of a magnetic field, we can write again the expression for the uniform component of the velocity as

$$
v_{0}=\min _{n}\left[\frac{\hbar}{m_{e} R}\left(n-\frac{\Phi}{\Phi_{0}}\right)\right] .
$$

Combining this result with Eqs. (26) and (27) we arrive at the dispersion relation for plasma oscillations in the ring (Fig. 4)

$$
\omega^{2}=\frac{N_{s} e^{2} \nu^{2} S}{m_{e} R^{2}}\left(1-\frac{3 \hbar^{2}}{m_{e}^{2} R^{2} v_{c}^{2}} \min _{n}\left[\left(n-\frac{\Phi}{\Phi_{0}}\right)^{2}\right]\right) \ln \left(\frac{\nu^{2}+\nu_{c}^{2}}{\nu^{2}}\right) .
$$

For small wave numbers $\nu \ll \nu_{c}$ the frequency of the plasma oscillation $\omega$ can be approximated by

$$
\omega \sim(\nu / R) c(\nu)
$$


where $c(\nu)$ is the velocity of the plasmons showing an AB behavior, expressed as a periodic function of the magnetic flux,

$$
c(\nu)=\sqrt{\frac{2 N_{s} e^{2} S}{m_{e}}\left(1-\frac{3 \hbar^{2}}{m_{e}^{2} R^{2} v_{c}^{2}} \min _{n}\left[\left(n-\frac{\Phi}{\Phi_{0}}\right)^{2}\right]\right) \ln \left(\frac{\nu_{c}}{\nu}\right)} .
$$

The oscillation amplitude of the plasmon frequency in large rings $R>\xi$ is proportional to $(\xi / R)^{2}$, which is similar to the case of the plasma oscillations in a tube (see Sec. III.A).

Characteristic properties of the plasma oscillation frequencies in superconducting rings are illustrated in Figs. 4(a) and 4(b). In Fig. 4(a) we display the plasmon frequencies as a function of the mode number $\nu$ for different values of the dimensionless flux, $\Phi / \Phi_{0}$; the mode numbers are discrete because of the periodic boundary conditions in the ring. In accordance with Eq. (30) higher modes (larger $\nu$ ) correspond to higher frequencies [27]. Applied magnetic flux through the ring induces a circular persistent current, which reduces the effective concentration of superconducting electrons participating in the plasma oscillations, with a consequent lowering of the plasmon frequency. Since the induced current is a periodic function of the magnetic flux, the frequency of the plasma oscillations for each mode is also a periodic function of the magnetic flux with a period $h c / 2 e$ (see Fig, $4(\mathrm{~b})$ ).

\section{SUMMARY.}

In this paper we have studied collective charge density oscillations (plasmons) in superconducting microtubes and microrings. Using a simple two-fluid model for the superconductor, we derived the dispersion relation for plasmons in a cylindrical tube of radius $R$, i.e. the plasmon frequency $\omega$ as a function of $k R$. We have demonstrated that depending on the magnitude of $k R$, a crossover emerges where the plasmon dispersion relation changes from a linear dependence on $k R$ (Eq. (21), the 1D limit) to a square root dependence (Eq. (22, the $2 \mathrm{D}$ limit). The behavior in these limiting cases is in agreement with previous theoretical predictions [5, 9] and experimental observations [10, 11, 12].

We have also considered the effects of weak magnetic fields on charge density excitations in superconducting microtubes and microrings, and we have shown that the dispersion relations for the plasmons are oscillatory functions of the magnetic flux with a universal period of $h c / 2 e$, and an amplitude of the order of $(\xi / R)^{2}$. Such behavior of the plasmons in superconducting microstructures is a manifestation of the Aharonov-Bohm effect.

In conclusion we discuss briefly dissipation effects in our systems. Our model does not take into account dissipations due to variations of the order parameter. We considered very long wavelength plasma oscillations characterized by small amplitudes $|\delta \Delta| \ll|\Delta|$, and frequencies that are restricted by the superconducting gap frequency $(2 \Delta / \hbar)$. For these conditions dissipation due to variations of the order parameter $\sim|\partial \Delta / \partial t|^{2}$ is negligibly small.

The expressions given in (20) and (29) for the dispersion relations of plasmons in superconducting tubes and rings are written for cases where the normal conductivity of the superconductors can be neglected. In general, a nonzero normal conductivity $\sigma_{n}$ adds to the dispersion relation an imaginary term which expresses energy dissipation and decay of the plasma oscillations. For example, for superconducting tubes one can write $\left(\bar{\omega}=\omega\left(\sigma_{n}=0\right)\right)$

$$
\omega\left(\sigma_{n}\right) \approx \bar{\omega}+i \gamma
$$


where for the mode with $m=0$ the ratio of the imaginary part of the frequency to the real part is

$$
\gamma / \bar{\omega} \sim\left(\sigma_{n} / \omega_{s}\right) k\left[R d I_{0}(k R) K_{0}(k R)\right]^{1 / 2}
$$

For the limiting case of a thin wire (1D), $k R \ll 1$, Eq. (33) gives $\gamma / \bar{\omega} \sim$ $\left(\sigma_{n} / \omega_{s}\right) k \sqrt{R d \ln (1 /(k R))}$. In the case of a thin film $(2 \mathrm{D}), k R \gg 1$, damping effects are described by the expression $\gamma / \bar{\omega} \sim\left(\sigma_{n} / \omega_{s}\right) \sqrt{k d}$, in agreement with the results of Ref. [5].

The plasmon damping, given by Eq. (33) is small for dirty superconductors $\left(\sigma_{n} \rightarrow 0\right)$. Higher normal conductivity results in stronger dissipation and decay of the plasma oscillations. This counterintuitive result can be explained qualitatively by the following arguments. When the collision time is small the coherent motion of the plasma waves is created by the superconducting electrons, whereas normal electrons are only partly involved in this motion, and the faster they achieve equilibrium by collisions the better they follow the collective motion of the other electrons in the plasma wave, and as a result the system evolves more adiabatically with less dissipation.

Acknowledgement: This study was supported by the U.S. Department of Energy, Grant No. FG05-86ER45234. 


\section{FIGURE CAPTIONS}

FIG. 1: A schematic of a superconducting cylinder and a ring. The width, $d$, of the cylindrical shell (a) and the diameter, $d$, of the wire from which the ring (b) is made of, are taken to be small, i.e. $d \ll R$ and $d \ll \xi$, where $\xi$ is the correlation length of the superconductor.

FIG. 2: The dispersion relation $(\hbar \omega / \Delta$ versus $k R$ ) for longitudinal plasmons in a superconducting cylinder. Here $\Delta$ is the energy gap for electron-hole-like excitations inside the superconductor, for which we use an estimate (near $T_{c}$ ) $\Delta \sim k_{B} \sqrt{T_{c}\left(T_{c}-T\right)}$ [23]. For long wavelength $(k R<1$, see inset) plasmons, the dispersion relation is approximately linear, whereas for plasmons with a shorter wavelength, $k R>1$, the dispersion relation is similar to the one for plasmons in a thin superconducting film $(\omega \sim \sqrt{k})$. The three different curves in the figure correspond to three different currents through the tube with $I_{1}<I_{2}<I_{3}$, with the current $I$ expressed in terms of the critical current, $\eta=I / I_{c}$. For each value of the current, we have found from Eq. (77) the corresponding value of the uniform background velocity $u_{\theta}$ and then substituted it in Eq. (20). Since higher velocities of the superconducting electrons correspond to lower effective concentrations $N_{s}^{e f f}$, the frequency of the plasma oscillations is lower for the larger currents through the tube.

FIG. 3: Aharonov-Bohm oscillations ( $\omega$ vs the dimensionless flux, $\Phi / \Phi_{0}$ ) of the dispersion relation for plasmons in a thin superconducting cylindrical tube. The parameters of the hollow tube are the following: radius $R=5 \times 10^{-4} \mathrm{~cm}$, width of the wall $d=10^{-7} \mathrm{~cm}$; The tube is made from niobium $\left(T_{c}=9.3 K\right)$, and for the order parameter $\Delta$ we use the same estimate as in Fig. 2. Both figures correspond to plasmons in the zero circular mode $m=0$ and a longitudinal wave vector $k=0.005 / R$. In (a) we show the frequency $\omega$ of the plasma oscillations as a function of the dimensionless magnetic flux for a fixed temperature $T=8.5 \mathrm{~K}$, for different values of the parameter $\eta=I / I_{c}$, where $I$ is the current along the tube, and $I_{c}$ is the critical current. In (b) we show for a fixed current along the tube $\left(I=0.1 I_{c}\right)$ the frequency of the plasma oscillations as a function of the dimensionless magnetic flux, for a range of temperatures.

FIG. 4: Frequencies of plasma oscillations in a superconducting ring. The results are shown for a niobium ring with a radius $R=2 \times 10^{-3} \mathrm{~cm}$. The cross sectional area of the wire (from which the ring is made from) is $S_{0}=5 \times 10^{-14} \mathrm{~cm}$. (a) The frequency $\omega$ for different values of $\Phi / \Phi_{0}$ (the magnetic flux in units of the flux quantum), as a function of the mode

number $\nu$. (b) The frequency $\omega$ as a function of the dimensionless flux, $\Phi / \Phi_{0}$, plotted for different values of the wave vector $\nu$, illustrating oscillatory behavior. 
[1] R.V. Carlson and A.M. Goldman, Phys. Rev. Lett. 34, 11 (1975).

[2] A. Schmid and G. Schön, Phys. Rev. Lett. 34, 941 (1975).

[3] S.N. Artemenko and A.F. Volkov, Zh. Eksp. Teor. Fiz. 69, 1764 (1975) [Sov. Phys. JETP 42, 896 (1975)].

[4] Since the phase velocity of the Carlson-Goldman (CG) mode is much smaller than the phase velocity of the acoustic plasmon mode, these two modes occur at very different wave vector ranges. Although both the acoustic and the CG type of excitations may exist in superconducting tubes and rings simultaneously, the CG mode has much smaller wavelengths. Because of the different nature of these modes the interaction between them can be neglected. Consideration of the influence of the Little-Parks effect on the CG mode in tubes and rings is left for a future study.

[5] I.O. Kulik Zh. Eksp. Teor. Fiz. 652016 (1973) [Sov. Phys. JETP 38, 1008 (1974)].

[6] R. A. Ferrell, Phys. Rev. Lett. 13, 330 (1964).

[7] E.N. Bogachek and G.A. Gogadze Zh. Eksp. Teor. Fiz. 67, 621 (1974) [Sov. Phys. JETP 40, 306 (1975)].

[8] For 2D behavior see T. Ando, A.B. Fowler, and F. Stern, Rev. Mod. Phys. 54, 437 (1982), and references therein.

[9] J.E. Mooij and G. Schön, Phys. Rev. Lett. 55, 114 (1985).

[10] O. Buisson, P. Xavier, and J. Richard, Phys. Rev. Lett. 73, 3153 (1994).

[11] F.J. Dunmore, D.Z. Liu, H.D. Drew, and S. Das Sarma, Phys. Rev. B 52, R731 (1995).

$[12]$ B. Caramota, F. Parage, F. Balestro, P. Delsing, and O. Buisson, Phys. Rev. Lett. 86, 480 (2001).

[13] N. Byers and C.N. Yang, Phys. Rev. Lett. 7, 46 (1961).

[14] W.A. Little and R.D. Parks, Phys. Rev. Lett. 9, 9 (1962).

[15] E.N. Bogachek, G.A. Gogadze, and I.O. Kulik, Phys. Stat. Sol. B 67, 287 (1975).

[16] Y. Aharonov and D. Bohm, Phys. Rev. 115, 485 (1959).

[17] S. Olariu and I.I. Popescu, Rev. Mod. Phys. 57, 339 (1985).

[18] Note that in normal mesoscopic conductors the AB effect for collective excitations was studied in Ref.[7] for quantum electromagnetic waves, and in E.N. Bogachek, I.V. Krive, I.O. Kulik, and A.S. Rozhavsky, Phys. Rev. B 42, 7614 (1990), for charge density wave systems.

[19] Dimensionality crossovers of the behavior of plasmons in normal carbon nanotubes were discussed by C. Yannouleas, E.N. Bogachek, and U. Landman, Phys. Rev. B 53, 10225 (1996).

[20] We follow the approach that was used in Ref. 5 for studying plasma waves in thin superconducting thin wires and films.

[21] J. Bardeen, Phys. Rev. Lett. 1, 399 (1958)

[22] G. Rickayzen, Proc. Phys. Soc. 89, 129 (1966)

[23] P.G. De Gennes, Superconductivity of metals and alloys, W.A. Benjamin, New York (1966).

[24] R. Tidecks, Current-induced nonequilibrium phenomena in quasi-one-dimensional superconductors, Springer-Verlag, Berlin (1990)

[25] J. Rubinstein, P. Sternberg, and Q. Ma, Phys. Rev. Lett. 99, 167003 (2007).

[26] Y. Liu, Yu. Zadorozhny, M. M. Rosario, B.Y. Rock, P. T. Carrigan, H. Wang, Science 294, 2332 (2001).

[27] Compare with the similar behavior of plasmon frequencies in carbon nanotubes, see e.g. Ref. 
in [19]. 


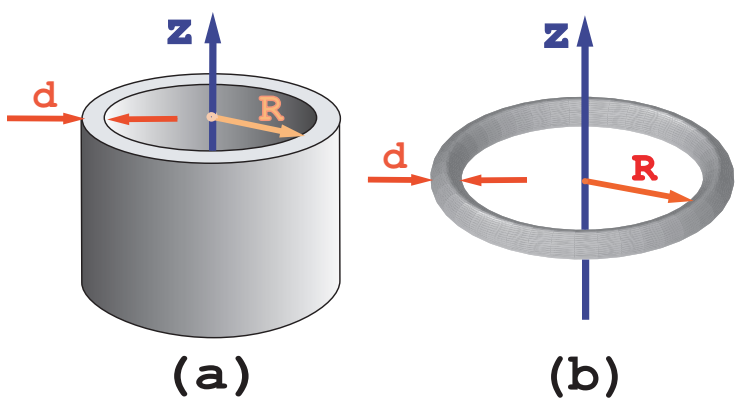

FIG. 1

FIG. 1: 


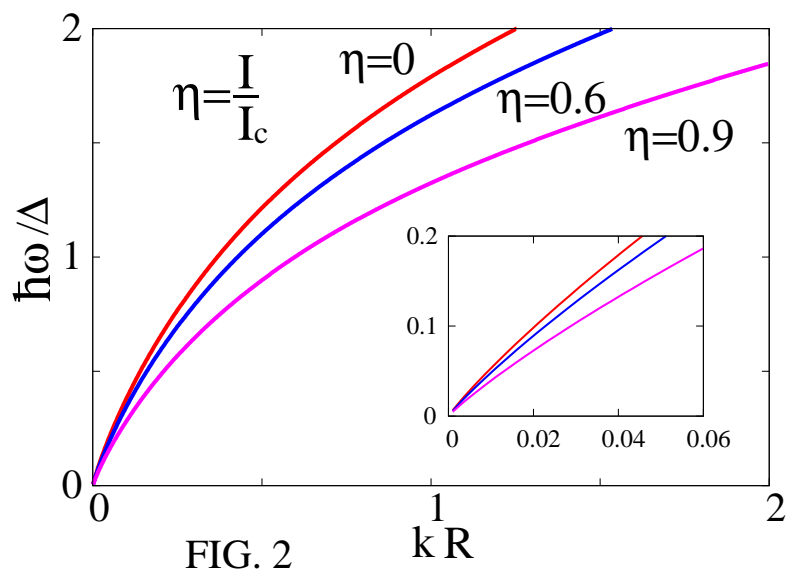

FIG. 2: 

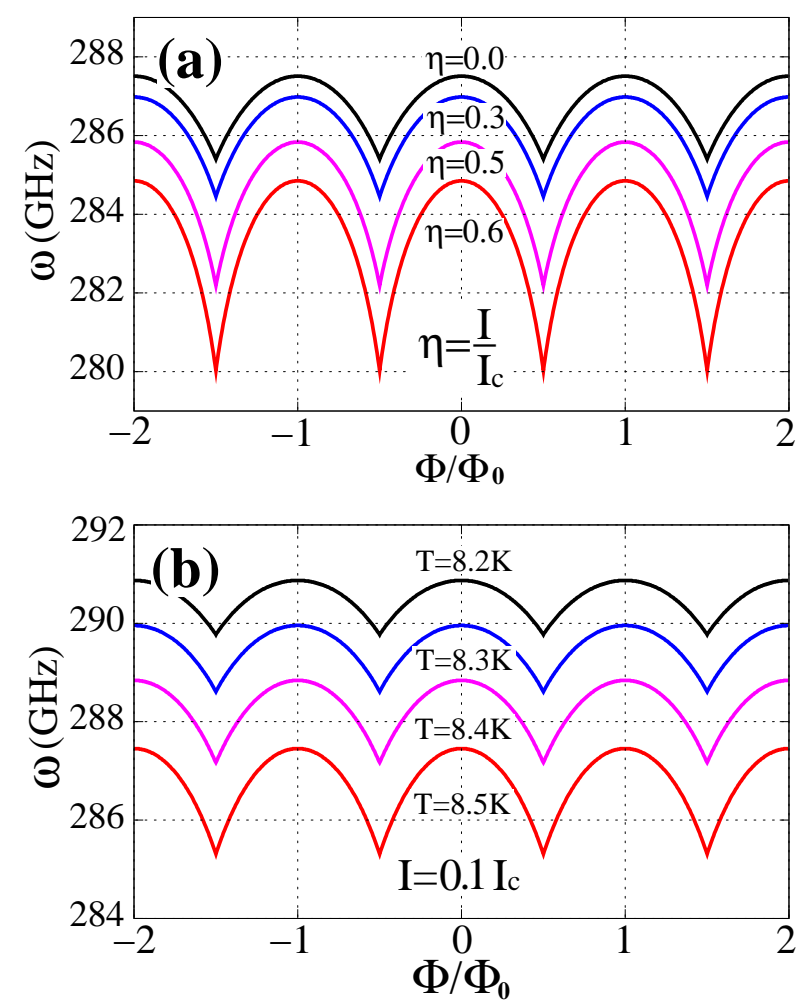

FIG. 3

FIG. 3: 

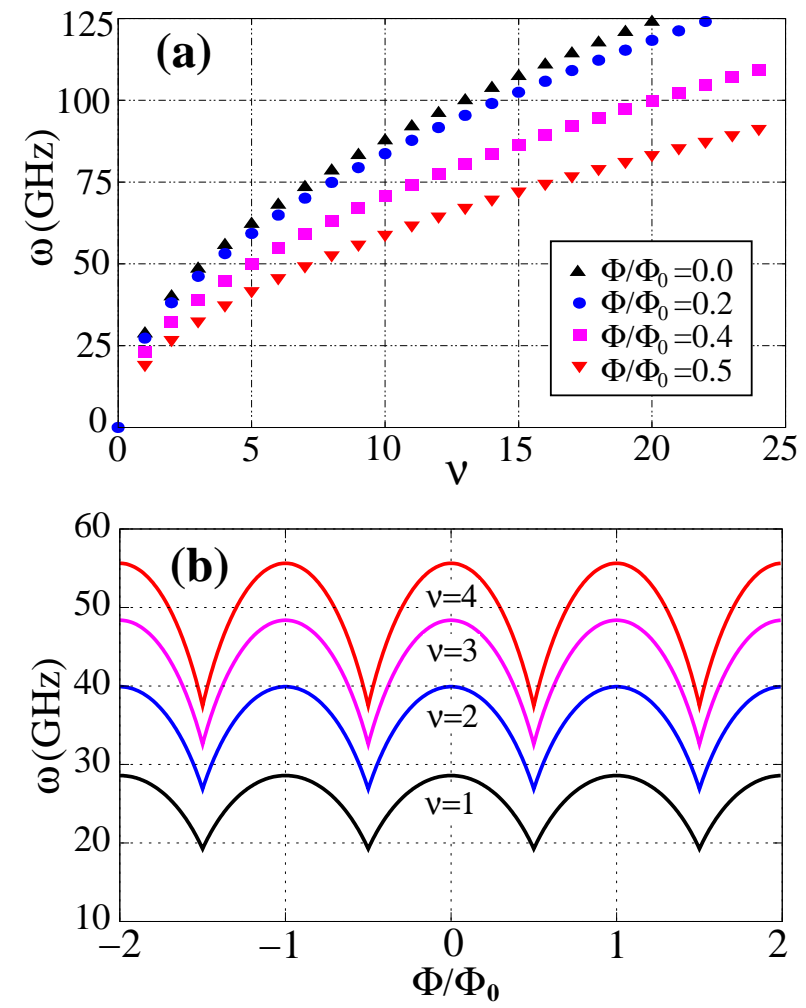

FIG. 4

FIG. 4: 